\title{
Recomendação de códigos da CID-10 para vigilância de reações adversas e intoxicações a medicamentos
}

\author{
Recommendation of ICD-10 codes for surveillance \\ of adverse drug reactions and drug intoxication
}

Daniel Marques Mota ${ }^{1}$

Álvaro Vigo ${ }^{1}$

Ricardo de Souza Kuchenbecker ${ }^{1}$

${ }^{1}$ Programa de PósGraduação em

Epidemiologia, Faculdade

de Medicina, Universidade

Federal do Rio Grande do

Sul. R. Ramiro Barcelos

2.400/2 , Rio Branco. 90035-

003 Porto Alegre RS Brasil.

dmarques2003@

yahoo.com.br

\begin{abstract}
ICD-10 is the 10th revision of the International Statistical Classification of Diseases and Related Health Problems (ICD), a medical classification list by the World Health Organization. It contains codes for diseases, signs and symptoms, abnormal findings, complaints, social circumstances, and external causes of injury or diseases. Associations between variables were evaluated using Pearson's chi-squared test and multiple correspondence analysis. Six hundred and ninety-one (691) codes were identified related to adverse drug reactions (52.1\%) and drug poisoning (47.9\%). A total of 687 (99.4\%) and 511 (73.9\%) codes were validated in 1 st and 2 nd validation, respectively. There were statistically significant differences ( $p$ $<0.05)$ between adverse reactions and drug poisoning in the variables used to characterize the reference list. The association between drug and hospital admission and death was statistically significant when stratified by type of adverse event ( $p$ $<0.001)$. Three groupings of codes were identified in multiple correspondence analysis where there are associations between categories of response assessed. The reference list can be a useful tool in pharmacovigilance actions in Brazil.
\end{abstract}

Key words International Classification of Diseases, Drug-related side effects and adverse reactions, Poisoning
Resumo Propor uma lista-referência de códigos da Classificação Internacional de Doenças e Problemas Relacionados à Saúde (CID-10) para a vigilância de reações adversas e intoxicações a medicamentos, denominados de eventos adversos. A elaboração da lista-referência percorreu quatro fases: definição dos códigos, validação e duas de caracterização da lista. As associações entre variáveis foram avaliadas por qui-quadrado de Pearson e análise de correspondência múltipla. Foram identificados 691 códigos relacionados com reação adversa a medicamentos $(52,1 \%)$ e intoxicação medicamentosa (47,9\%). Um total de 687 $(99,4 \%)$ e 511 (73,9\%) códigos foram validados na $1^{a}$ e $2^{a}$ validação, respectivamente. Há diferenças estatisticamente significativas $(p<0,05)$ entre reações adversas e intoxicação medicamentosa nas variáveis utilizadas para a caracterização da lista -referência. A associação entre medicamento e admissão hospitalar e óbito foi estatisticamente significativa quando estratificada por tipo de evento adverso $(p<0,001)$. Na análise de correspondência múltipla identificou-se três agrupamentos de códigos em que há associações entre as categorias de resposta das variáveis estudadas. A lista-referência pode ser uma ferramenta útil nas ações de farmacovigilância no Brasil.

Palavras-chave Classificação Internacional de Doenças, Efeitos colaterais e reações adversas relacionados a medicamentos, Envenenamento 


\section{Introdução}

A farmacovigilância é definida como ciência e atividades relativas à detecção, avaliação, compreensão e prevenção de reações adversas ou quaisquer outros possíveis problemas relacionados com medicamentos ${ }^{1}$. Colabora com a segurança do paciente e coletividade ao promover a vigilância dos eventos adversos a medicamentos, incluíndo as reações adversas a medicamentos (RAM) e intoxicações medicamentosas (IM), principalmente aquelas consideradas graves, como as que resultam em admissões hospitalares e óbitos. Sua principal fonte de dados tem sido os sistemas de informações baseado em notificações espontâneas de eventos adversos ${ }^{2}$, cujas limitações ${ }^{3}$ têm estimulado a busca de fontes adicionais para a vigilância de RAM e IM.

As bases de dados administrativas (ou não) oriundas de outros sistemas de informações em saúde apresentam várias vantagens para uso na farmacovigilância ${ }^{4}$. Têm sido utilizadas para descrever e analisar padrões e tendências de RAM e IM, que resultaram em admissões hospitalares e óbitos $^{5-9}$. Esses eventos podem ser identificados por códigos de diagnóstico da Classificação Estatística Internacional de Doenças e Problemas Relacionados à Saúde (CID) da Organização Mundial de Saúde (OMS), décima versão (CID$10)^{10}$. Este estudo objetivou propor uma lista-referência de códigos da CID-10 para vigilância de RAM e IM a ser utilizada em estudos realizados no Brasil.

\section{Método}

Para efeitos deste estudo, alguns termos com suas respectivas definições e observações foram empregados: (1) Reação(ões) adversa(s) a medicamento(s) (RAM) - toda resposta nociva e não intencional a um medicamento relacionada a qualquer dose normalmente utilizada em seres humanos para profilaxia, diagnóstico e tratamento de doenças ou modificação de funções fisiológicas ${ }^{1}$ Os termos "reação adversa" e "efeito adverso" foram considerados sinônimos; (2) Intoxicação(ões) medicamentosa(s) (IM) - exposição de um indivíduo (por ingestão, injeção ou inalação) a uma quantidade de medicamento(s) que potencialmente pode $(\mathrm{m})$ ocasionar danos significativos à saúde ${ }^{11,12}$. Os termos "intoxicação" e "envenenamento" foram considerados sinônimos. A expressão "droga(s)" descrita nos códigos da CID-10 foi definida como sinônimo de "medicamento(s)". A exceção ocorreu para os códigos relacionados às drogas de uso considerado como ilícito. Neste estudo, os tipos de eventos adversos incluidos foram as RAM e IM.

A CID-10 foi a fonte de dados utilizada para a obtenção dos códigos de diagnóstico relacionados com RAM e IM. Trata-se de ferramenta para o diagnóstico das causas de mortalidade e morbidade, utilizada por diferentes sistemas de vigilância e assistência de muitos países ${ }^{13,14}$. Oferece nomenclatura padronizada baseada em códigos alfa-numéricos que permite melhorar a concordância e confiabilidade diagnóstica em diferentes locais $^{10,15}$.

A elaboração da lista de códigos da CID-10 relacionados com RAM e IM, foi realizada por um médico e um farmacêutico com experiência em farmacoepidemiologia e farmacovigilância e ocorreu no período de novembro de 2014 a maio de 2015. Qualquer divergência nos procedimentos adotados foi resolvida por meio de consenso. A elaboração da lista-referência foi realizada em quatro fases descritas a seguir.

$1^{a}$ fase. Definição dos códigos

A definição obedeceu a exame minucioso da CID-10, em formato físico e eletrônico, e, também, da lista de códigos do estudo de revisão sistemática publicada por Hohl et al. ${ }^{16}$, considerado como padrão de referência para este estudo. Foram identificados todo e qualquer código, em nível de subcategorias, compatível com um diagnóstico de RAM e IM. As categorias também foram consideradas na composição da lista-referência. $\mathrm{O}$ exame foi composto por três etapas abaixo descritas.

A $1^{\text {a }}$ etapa envolveu a realização de consultas, de forma manual, ao dicionário da CID-10 no formato em papel, volume 1, no idioma português ${ }^{10}$, produzindo uma primeira lista. Essa lista foi conferida e complementada por meio de pesquisa no programa eletrônico PesqCID, versão 2.4 do Departamento de Informática do SUS do Ministério da Saúde (Datasus/MS), utilizando as palavras truncadas "medicament ${ }^{*}$ ", "drog*”, "vacin*", "envenenament $t^{*}$, "intoxicaç ", "efeit", advers $^{\star}$ " e "reaç $c^{\star}$ ". Esta 2a etapa objetivou capturar novos códigos ou confirmar aqueles selecionados na $1^{\text {a }}$ etapa.

Nas $1^{\text {a }}$ e $2^{\text {a }}$ etapas, os códigos selecionados para a composição da lista-referência obedeceram aos seguintes requisitos: a) códigos que identificavam o medicamento como o agente causador do evento adverso; b) códigos que identificavam o medicamento pelo menos como um dos agentes causadores do evento adverso; c) códigos que, 
embora não atenderam aos itens "a" e "b", apresentavam alguma nota adicional sobre o potencial envolvimento de medicamentos e natureza do evento adverso; e d) códigos que, a partir do conhecimento prévio dos autores, identificavam a doença com etiologia relacionada a medicamento, como Síndrome de Reye e agranulocitose.

A lista de códigos do estudo de revisão sistemática ${ }^{16}$ foi utilizada para a atualização da segunda lista, dando origem à $3^{a}$ e última etapa do processo de definição dos códigos da lista-referência. A escolha deste estudo foi motivada por duas razões: i) tratar de uma revisão sistemática atualizada de artigos científicos que utilizaram códigos da CID-10 para identificar RAM e IM responsáveis por admissões hospitalares e óbitos em bases de dados; e ii) acesso à lista, contendo 608 códigos.

$\mathrm{Na} 3^{\mathrm{a}}$ etapa, além dos requisitos referidos nas etapas anteriores, outras regras foram adotadas para a inclusão de novos códigos. As regras, contempladas de forma simultânea, foram: i) o código deve ser classificado em uma das categorias $\mathrm{A} 1, \mathrm{~A} 2, \mathrm{~B} 1, \mathrm{~B} 2, \mathrm{~V}, \mathrm{C}$ e D estabelecidas pelos autores do estudo (Quadro 1). As categorias E e $U$ foram desconsideradas devido à pouca ou nenhuma possibilidade dos eventos adversos estarem relacionados a medicamentos ${ }^{16}$; e ii) o código deve constar em cinco ou mais artigos científicos identificados no estudo de revisão sistemática ${ }^{16}$. A quantidade de artigos, definida de forma aleatória, teve o propósito de melhor identificar a validação dos códigos da lista-referência. Salienta-se ainda que antes da inserção definitiva na lista, cada código que atendeu a ambas as regras foi reavaliado em relação a notas adicionais contidas no próprio código por meio de pesquisa no PesqCID.

$2^{a}$ fase. Validação de códigos repetidos

Compreendeu o processo de verificação de códigos que, uma vez presentes na lista-referência, também foram identificados em estudos científicos já publicados. Ocorreu em dois momentos. O primeiro consistiu na checagem dos códigos da lista-referência com aqueles presentes nas listas de estudos científicos. As publicações selecionadas foram o estudo de revisão sistemática $^{16}$ e estudos publicados contendo dados brasileiros $^{5-9,17}$.

O segundo momento da validação baseouse na identificação de códigos que registraram RAM e IM responsáveis por admissões hospitalares e óbitos (desfechos) em estudos científicos. Os estudos selecionados foram oriundos da seção Referências do estudo de revisão sistemática, estudos com dados brasileiros e quatro estudos científicos identificados durante o processo de pesquisa bibliográfica.

Os artigos científicos mencionados na seção referências do estudo de revisão sistemática ${ }^{16}$ foram selecionados a partir de dois critérios de elegibilidade: i) título do artigo pesquisado na seção Referências. Na dúvida em selecionar ou não o estudo, prevalecia a seleção do mesmo; e ii) informações verificadas no resumo sobre admissão hospitalar e/ou óbito relacionadas com códigos da CID-10. Foram excluídos os estudos de validação de códigos (confrontam os códigos na base de dados com aqueles contidos nos prontuários médicos), artigos de discussão e análise do estado da arte (revisões narrativas). Durante a 2a validação da lista-referência, quatro estudos científicos recém-publicados e não referenciados no estudo de revisão sistemática foram identificados e incorporados ao estudo ${ }^{18-21}$.

As publicações científicas com dados brasileiros foram selecionadas por meio de consultas ao Google Acadêmico. Para isso, duas estratégias de busca foram definidas, de acordo com as bases de dados eletrônicas pesquisadas (Scielo e PubMed) e limitadas no tempo (1992 - ano de implantação da CID-10 - a março de 2015) e idiomas (português, inglês e espanhol). As estratégias de busca foram: scielo and reacao adversa and intoxicacao and medicamento and CID-10 and Brasil e pubmed and adverse drug event and ICD-10 and database and Brazil. Os estudos selecionados foram avaliados visando identificação de lista de códigos da CID-10.

Uma planilha eletrônica disponibilizada pelo Datasus/MS (http://www.datasus.gov.br/cid10/ V2008/descrcsv.htm) que indicava a probabilidade do código estar relacionado a óbito, também foi utilizada como um instrumento complementar no $2^{\circ}$ momento da validação dos códigos CID-10.

$3^{a}$ fase. Caracterização da lista-referência: natureza dos eventos e categorização dos códigos

Os códigos da lista-referência foram classificados segundo a natureza do evento adverso em RAM e IM, observando a descrição textual de cada código na CID-10 e sua adequação aos termos e definições estabelecidos. Para a categorização dos códigos foi utilizada uma adaptação do sistema definido por duas publicações científicas $^{22,23}$ e modificado por Hohl et al. ${ }^{16}$. Os códigos da lista-referência foram classificados em sete categorias, conforme exemplificado no Quadro 1.

$4^{a}$ fase. Caracterização da lista-referência: definição do nexo causal dos códigos 
Quadro 1. Caracterização dos códigos da lista-referência: uma adaptação a partir de artigos publicados na literatura científica ${ }^{19-21}$.

\begin{tabular}{|c|c|c|c|c|}
\hline \multirow{2}{*}{$\begin{array}{l}\text { Categorias } \\
\text { dos códigos }\end{array}$} & \multirow{2}{*}{ Definição } & \multirow{2}{*}{$\begin{array}{c}\text { Natureza } \\
\text { do EAM }\end{array}$} & \multicolumn{2}{|r|}{ Exemplos } \\
\hline & & & Código & Descrição do código \\
\hline A1 & $\begin{array}{l}\text { A causalidade foi estabelecida } \\
\text { entre medicamento e reação } \\
\text { adversa na CID- } 10 \text {. }\end{array}$ & RAM & $\mathrm{J} 70.2$ & $\begin{array}{l}\text { Transtornos pulmonares intersticiais } \\
\text { agudos, induzidos por droga. }\end{array}$ \\
\hline A2 & $\begin{array}{l}\text { A causalidade foi relacionada } \\
\text { com medicamento e outras } \\
\text { causas CID- } 10 \text {. }\end{array}$ & RAM & I 42.7 & $\begin{array}{l}\text { Cardiomiopatia devida a drogas e } \\
\text { outros agentes externos. }\end{array}$ \\
\hline B1 & $\begin{array}{l}\text { A causalidade foi estabelecida } \\
\text { entre medicamento e intoxicação } \\
\text { medicamentosa na CID- } 10 \text {. }\end{array}$ & IM & T36 & $\begin{array}{l}\text { Intoxicação por antibióticos } \\
\text { sistêmicos. }\end{array}$ \\
\hline B2 & $\begin{array}{l}\text { A causalidade foi relacionada } \\
\text { com medicamento e outra causa } \\
\text { na CID- } 10 .\end{array}$ & IM & $\mathrm{X} 44$ & $\begin{array}{l}\text { Envenenamento [intoxicação] } \\
\text { acidental por exposição a outras } \\
\text { drogas, medicamentos e substâncias } \\
\text { biológicas não especificadas. }\end{array}$ \\
\hline $\mathrm{C}$ & $\begin{array}{l}\text { O evento muito provavelmente } \\
\text { foi causado por um } \\
\text { medicamento. }\end{array}$ & RAM & L51.2 & $\begin{array}{l}\text { Necrólise epidérmica tóxica } \\
\text { [Síndrome de Lyell]. }\end{array}$ \\
\hline $\mathrm{D}$ & $\begin{array}{l}\text { O evento foi causado } \\
\text { provavelmente por um } \\
\text { medicamento. }\end{array}$ & RAM & N17.0 & $\begin{array}{l}\text { Insuficiência renal aguda com } \\
\text { necrose tubular. }\end{array}$ \\
\hline V & $\begin{array}{l}\text { A causalidade foi estabelecida } \\
\text { entre medicamento e reação } \\
\text { adversa na CID-10. }\end{array}$ & RAM & A80.0 & $\begin{array}{l}\text { Poliomielite paralítica aguda, } \\
\text { associada ao vírus vacinal. }\end{array}$ \\
\hline
\end{tabular}

Na definição do nexo causal dos códigos, os autores assumiram duas premissas: 1) o nexo causal entre medicamento e evento adverso dos códigos classificados nas categorias A1, A2, B1, B2 e V fundamentou-se na avaliação diagnóstica feita por médicos diretamente com o paciente. Essa avaliação foi, presumidamente, baseada em evidências suficientes relacionadas a pelo menos os seguintes elementos: i) sinais e sintomas que caracterizaram o evento adverso, história de uso de medicamento ou outra substância feito pelo paciente; e ii) temporalidade compatível, ou seja, a causa precedeu o efeito; e 2) o nexo causal entre medicamento e evento adverso dos códigos das categorias $\mathrm{C}$ e D foi definido pelos autores a partir de um julgamento clínico-epidemiológico, baseado no evento adverso identificado, informações adicionais advindas do conhecimento prévio dos autores e relação temporal desconhecida. Esta última premissa foi, também, fundamentada por dois estudos científicos ${ }^{24,25}$.

A partir das premissas, os autores estabeleceram uma escala qualitativa para a definição do nexo causal dos códigos da lista-referência, utilizando, para isso, alguns termos internacionalmente aceitos para a avaliação da causalidade de eventos adversos em farmacovigilância (Figura 1) 24-26.

Outras variáveis utilizadas para caracterizar os códigos da lista-referência foram: capítulos e categorias da CID-10, sexo (masculino, feminino e sem restrição de sexo) e desfecho, prevendo as seguintes alternativas: admissão hospitalar, óbito, admissão hospitalar e óbito, admissão hospitalar/ pouca possibilidade de ocasionar óbito, pouca possibilidade de ocasionar óbito e sem registros de defechos.

As associações entre variáveis foram avaliadas por meio da estatística qui-quadrado de Pearson $\left(\chi^{2}\right)$ e análise de correspondência múltipla, visando identificar alguma relação entre os códigos de RAM e IM estabelecidos como "definido" quanto ao nexo causal e suas possíveis consequência em termos de desfechos estabelecidos no estudo. Por exemplo, os códigos avaliados como "definido" estão associados com admissão hospitalar, óbito ou ambos?

A associação entre medicamento (variável explicativa) e admissão hospitalar/óbito (variável dependente) foi medida pela diferença percentual de linhas e colunas da tabela $2 \times 2$. A variável 


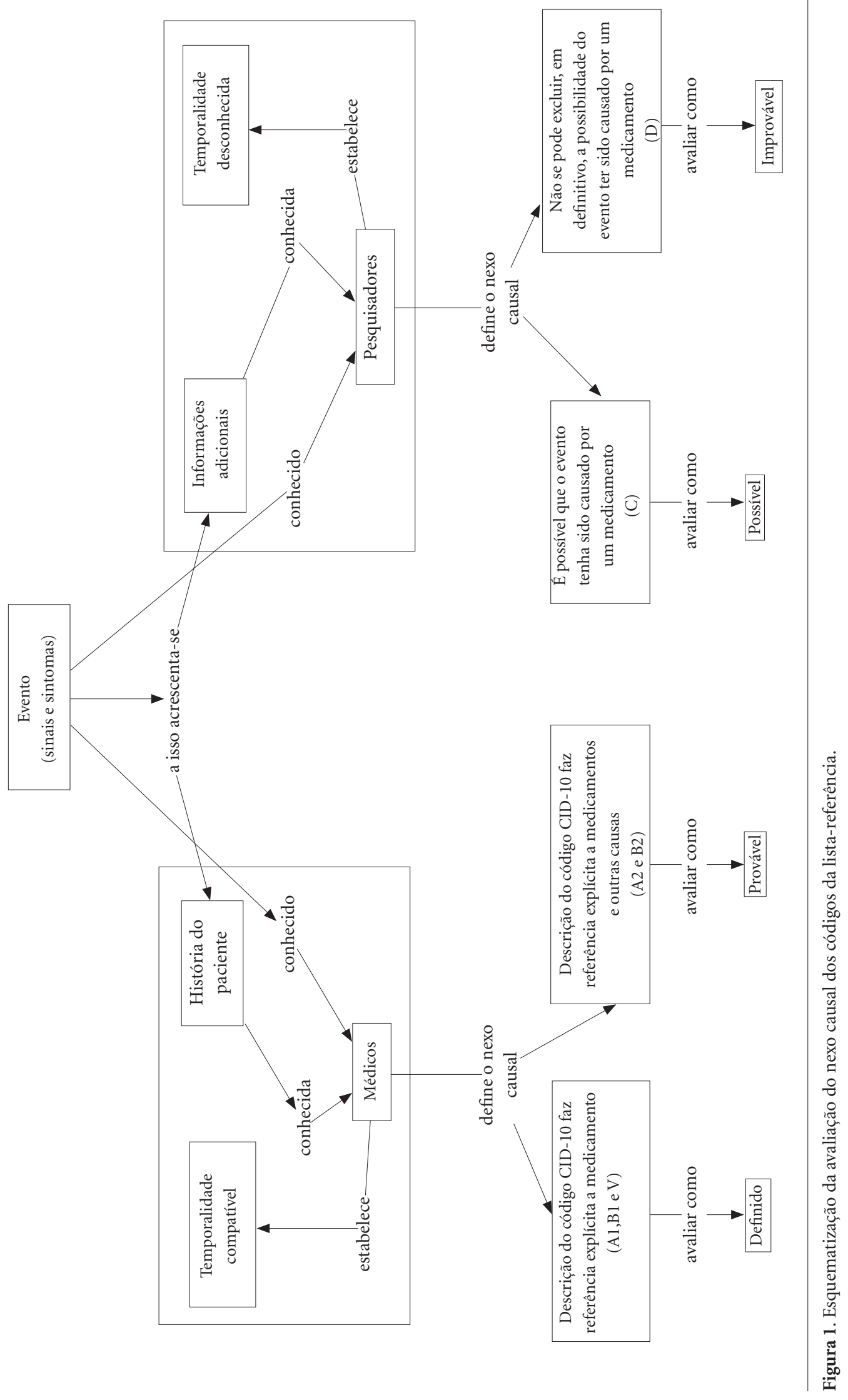


dependente foi composta pelo conjunto de códigos com registros de casos de admissão hospitalar e óbito (desfechos). A variável "nexodef" foi utilizada com proxy da variável explicativa. Esta variável estabelece o nexo causal dos códigos em duas categorias de resposta: "definido" ou "não definido". Os códigos classificados como "definido" são aqueles em que o medicamento é o único agente causador do evento adverso. Uma terceira variável, "tipo de evento adverso (RAM e IM)", foi utilizada como uma variável controle. O nível de $5 \%$ de significância foi utilizado nas análises.

$\mathrm{Na}$ análise de correspondência múltipla (ACM), as variáveis "categorização dos códigos", "desfechos" e "nexo causal" foram utilizadas na produção do gráfico, a partir do qual foi possível avaliar as relações entre as variáveis estudadas. A associação, ou não, entre as categorias de resposta das variáveis foi dada pela proximidade entre elas no gráfico, incluíndo os pontos dentro de um mesmo quadrante. A variável "tipo de evento adverso" foi adicionada à ACM como uma variável complementar. Nenhum dos pressupostos para a realização dessa técnica estatística foi violado ${ }^{27}$.

Um banco de dados foi organizado em planilha eletrônica (Microsoft Office Excel ${ }^{\circledR}$ ) em que cada código da CID-10 foi registrado em uma linha e cada variável de caracterização ocupou uma coluna da planilha. A análise estatística dos dados foi realizada no software SPSS, versão 18.03. O teste de aderência baseado na estatistica $\chi^{2}$ foi utilizado para verificar se as categorias de resposta de cada variável estavam uniformemente distribuídas. $\mathrm{O}$ teste de independência baseado na estatística $\chi^{2}$ foi utilizado para avaliar se as diferenças observadas entre RAM e IM nas variáveis estudadas foram estatisticamente significativas. Resíduos ajustados (RA) foram utilizados para verificar as associações locais entre as categorias de resposta das variáveis estudadas, considerando estatisticamente significativos os resíduos maiores que 1,96, em valores absolutos.

Este estudo é parte do projeto de pesquisa "Hospitalizações e óbitos por medicamentos e fatores associados à prescrição/venda: contribuições para estratégias de farmacovigilância no Brasil" aprovado pelo comitê de ética em pesquisa do Hospital de Clínicas de Porto Alegre da Universidade Federal do Rio Grande do Sul.

\section{Resultados}

A descrição dos processos de identificação e validação dos códigos da lista-referência com seus respectivos valores está apresentada na Tabela 1. O estudo produziu três listas-referência. $\mathrm{Na}$ primeira foram identificados 691 códigos relacionados com RAM $(52,1 \%)$ e IM $(47,9 \%)$; na segunda, 687 (99,4\%) códigos foram identificados nas listas de artigos científicos (1 $1^{a}$ validação); e na terceira lista-referência foram identificados 511 códigos que foram responsáveis por admissão hospitalar e/ou óbito ( $2^{a}$ validação). A lista -referência completa está apresentada nas Tabelas 1 e 2 .

Os novos códigos selecionados pelo PesqCID (2a etapa) para compor a lista-referência, corresponderam a sete, atendendo aos requisitos " $\mathrm{a}$ " (n =5) e "b" (n =2). Todos os códigos incluídos na lista-referência provenientes do estudo de revisão sistemática ( $3^{\mathrm{a}}$ etapa) enquadraram-se melhor no requisito "d", com a seguinte distribuíção por categorias: A2 $(\mathrm{n}=1)$; C $(\mathrm{n}=3)$ e D $(\mathrm{n}=10)$. A maioria dos códigos $(\mathrm{n}=10)$ foram citados por cinco estudos científicos. Os códigos da lista-referência nas categorias de resposta da variável "etapas de identificação dos códigos" não seguem uma distribuição equitativa, ou seja, as frequências observadas são diferentes das frequências esperadas $(\mathrm{p}<0,0001)$.

Em relação à $1^{\text {a }}$ validação de códigos, 596 $(86,2 \%)$ códigos da lista-referência constavam na lista do estudo de revisão sistemática (padrão de referência). Cinco estudos com dados brasileiros foram utilizados na $1^{\text {a }}$ validação de códigos, com os quais mais $78(11,3 \%)$ códigos foram validados. Restando, até então, 17(2,4\%) códigos não identificados após a finalização da $1^{\text {a }}$ validação de códigos. Durante a 2a validação, 13 códigos da lista-referência foram validados, os quais estavam presentes na lista de códigos do estudo publicado por Li et al. ${ }^{28}$. Este estudo fazia parte da seção Referências do estudo de revisão sistemática, sendo que tais códigos não constavam na lista do estudo considerado padrão de referência. Assim, apenas quatro códigos (K03.2, K22.1, O68 e Q73.1) não foram identificados na $1^{\text {a }}$ validação com os códigos das listas de estudos publicados na literatura.

$\mathrm{Na} 2^{a}$ validação foram utilizados 26 estudos científicos em que na seção de Resultados citavam algum código CID-10 que produziu admissão hospitalar e/ou óbito, dos quais três com dados brasileiros ${ }^{5,8,9}, 19$ oriundos do estudo de revisão sistemática ${ }^{22,23,28-44}$ e 4 estudos recém-publicados $^{18-21}$. Os $511(73,9 \%)$ códigos identificados na $2^{\text {a }}$ validação já haviam sido identificados na $1^{\text {a }}$ validação. Dos $180(26,0 \%)$ códigos que não contabilizaram registros de eventos adversos 


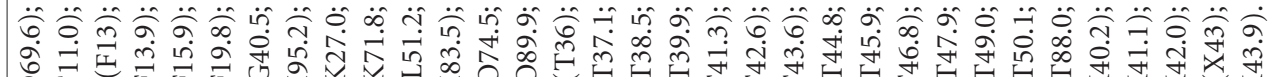

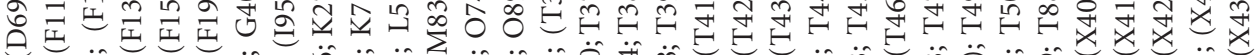

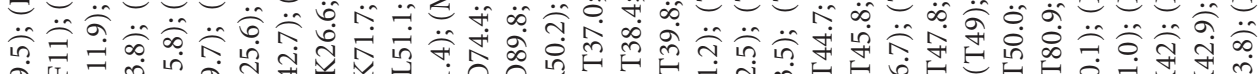

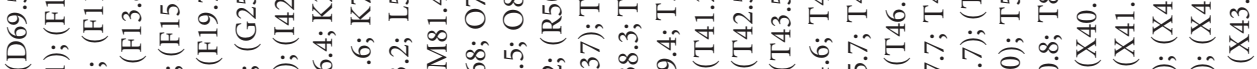

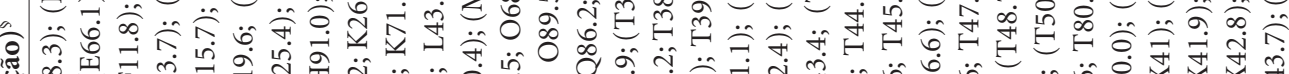

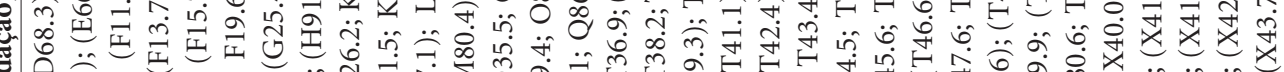

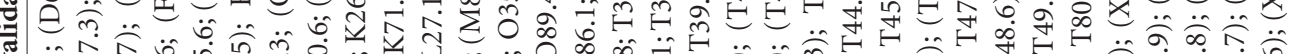
च đ̃

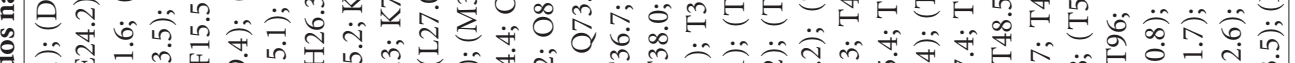

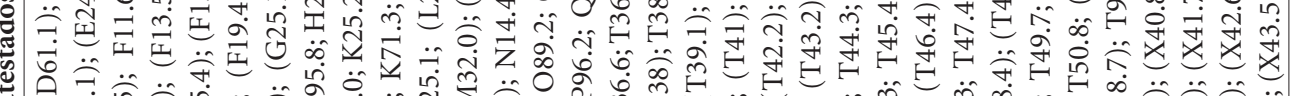
ప

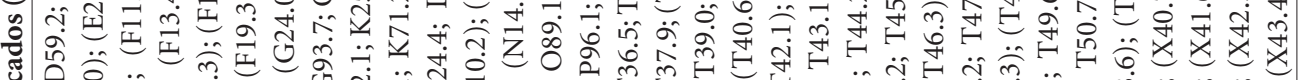

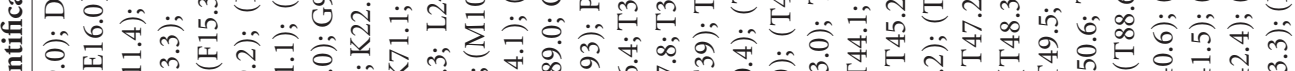

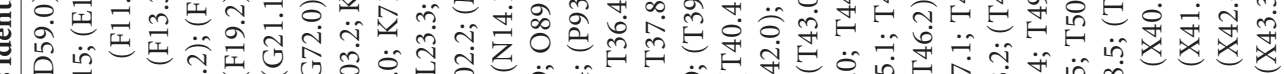

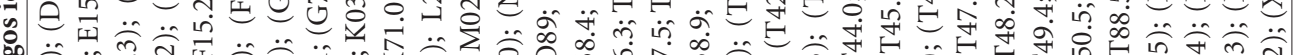

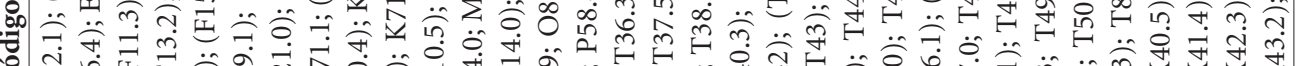

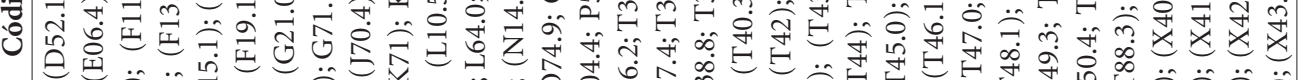

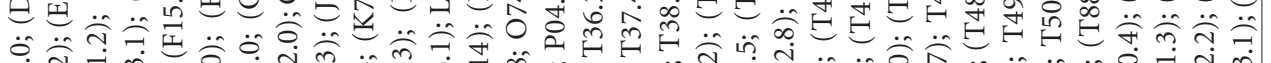

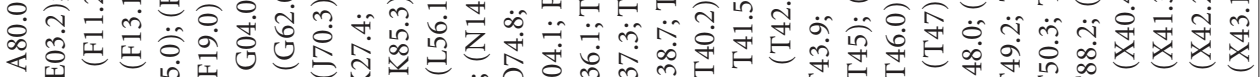

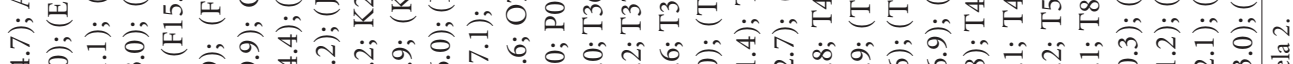

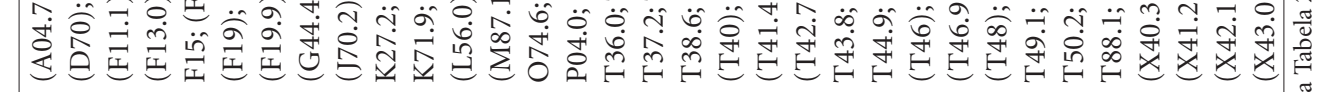

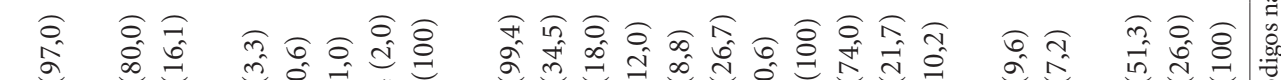

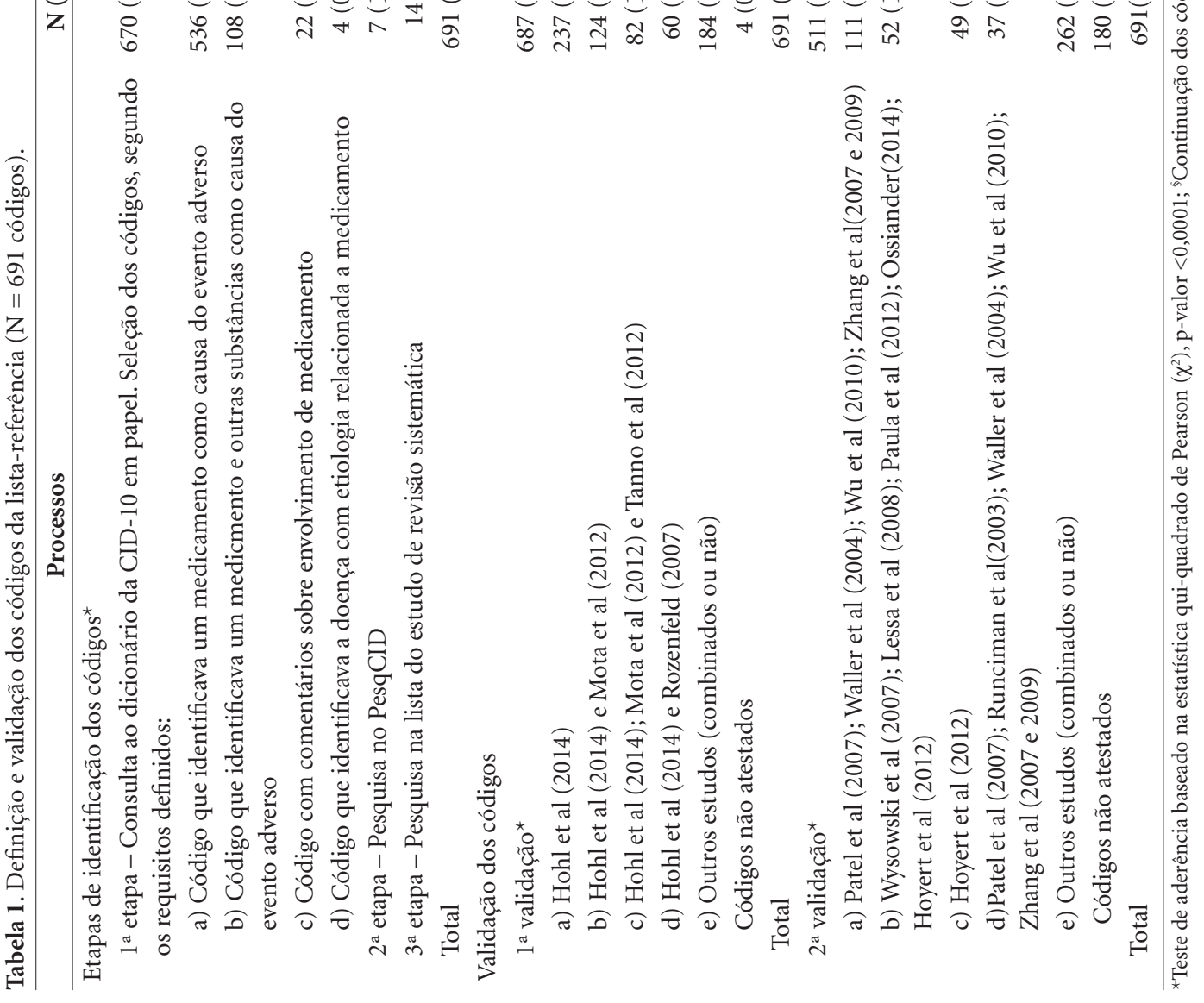


graves, 2 (8,8\%) deles (K22.1 e O68) não haviam sido identificados na $1^{\text {a }}$ validação. Não foi identificada uma distribuição uniforme dos códigos da lista-referência nas categorias das variáveis " 1 a e 2a validação dos códigos” ( $\mathrm{p}<0,0001)$ (Tabela 1).

Dos 180 códigos que não foram identificados na $2^{\text {a }}$ validação, pelo menos $22(12,2 \%)$ não seriam responsáveis por admissão hospitalar, pois teriam o potencial de ocorrer com o paciente já hospitalizado, restando o desfecho "óbito" e 9 (5,0\%) códigos apareceram com pouca probabilidade de ocasionar óbitos, segundo a planilha técnica do Datasus/MS.

A Tabela 2 apresenta uma caracterização geral dos 691 códigos que constam na lista-referência. Devido à descrição ou nota adicional na CID-10 dos códigos selecionados, 4 (0,6\%) deles, (P04.0, P93, T80.6 e Z03.6) podem receber dupla classificação: RAM e IM. No entanto, para as análises apresentadas neste estudo, os autores optaram em classificar os códigos em apenas um dos tipos de eventos adversos. Foram observadas diferenças estatisticamente significativas $(\mathrm{p}<0,05)$ entre RAM e IM em todas as variáveis estudadas (Tabela 2).

Todos os capítulos da CID-10 contidos na lista-referência apresentaram códigos classificados como RAM enquanto que aqueles definidos como IM foram encontrados em 4 (20,0\%) deles (Capítulos V, XI, XIX e XX). Nenhum dos códigos pertencentes aos capítulos II (Neoplasias) e XXII (Códigos para propósitos especiais) fez-se presente na lista-referência. Os valores dos resíduos ajustados indicam que há maior associação entre RAM e os capítulos V $(\mathrm{RA}=4.1), \mathrm{XV}(\mathrm{RA}=$ $4,0)$, VI $(\mathrm{RA}=3,8)$ e demais capítulos $(\mathrm{RA}=7,8)$, bem como entre IM e capítulo XIX $(\mathrm{RA}=13,0)$.

De um total de 131 categorias da CID-10 constantes na lista-referência, $99(75,6 \%)$ apresentaram códigos classificados como RAM e 36 $(27,4 \%)$ como IM. Onze foi o maior número de códigos contidos em uma categoria da CID-10. Esse valor foi observado em 37 (28,2\%) delas, sendo que na maior parte dessas categorias predominaram os códigos classificados como IM (n $=24 ; 64,9 \%$ ).

As categorias de resposta A1 e B1 apresentaram, juntas, o maior número de códigos da lista -referência ( $\mathrm{n}=429 ; 62,1 \%$ ) (Tabela 2$)$. Os valores dos resíduos ajustados indicam que há uma associação entre RAM e as categorias de resposta $\mathrm{A} 1(\mathrm{RA}=18,5), \mathrm{A} 2(\mathrm{RA}=8,5), \mathrm{D}(\mathrm{RA}=4,7)$ e V $(\mathrm{RA}=4,1)$, assim como entre IM e as categorias de resposta $\mathrm{B} 1(\mathrm{RA}=16,7)$ e $\mathrm{B} 2(\mathrm{RA}=13,4)$. A razão das categorias de resposta definido/provável da variável "nexo causal” entre os códigos classificados como RAM (3,7:1) é quase três vezes maior quando comparada à mesma razão entre aqueles classificados como IM $(1,4: 1)$. Há maior associação entre RAM e as categorias de resposta, definido e improvável, bem como entre IM e a categoria de resposta provável, segundo os valores dos resíduos ajustados. Na variável sexo, houve o predominio dos códigos de uso exclusivo feminino (10:1).

Observou-se que na variável "desfechos", três códigos identificados como relacionados a pouca possibilidade de causar óbito, apresentaram registros de casos de admissão hospitalar (H91.0, L56.0 e L56.1) (Tabela 2). As categorias de resposta da variável “desfechos” admissão hospitalar $(\mathrm{RA}=10,3)$ e pouca possibilidade de produzir óbito $(\mathrm{RA}=2,9)$ apresentaram maior associação com RAM, enquanto que as categorias de resposta ambos (admissão hospitalar e óbito) (RA $=8,0)$ e sem desfecho $(\mathrm{RA}=3,0)$ se mostraram associadas com IM, de acordo com os valores dos resíduos ajustados.

Não foi observada associação estatisticamente significativa entre medicamento (nexodef) e admissão hospitalar/óbito ( $\mathrm{p}=0,32)$. No entanto, verificou-se uma associação positiva entre tipo de evento adverso e admissão hospitalar/óbito ( $\mathrm{p}$ $=0,02$ ) e, também, entre Tipo de evento adverso e medicamento $(\mathrm{p}<0,001)$. Observou-se uma influência da variável-controle, "Tipo de evento adverso", sobre a associação entre medicamento e desfechos. A associação entre medicamento e admissão hospitalar/óbito foi estatisticamente significativa quando estratificada por "Tipo de evento adverso", classificado como RAM ( $\mathrm{p}<$ $0,001)$ e IM ( $\mathrm{p}<0,001)$, sendo que, neste último caso, a associação foi negativa.

$\mathrm{Na}$ análise de correspondência múltipla, as duas dimensões apresentaram valores de inércia muito próximos, sendo que o valor da dimensão 1 foi um pouco maior, explicando $77,0 \%$ da variabilidade da massa dos dados (Figura 2). Um exemplo na interpretação desse valor é: variações na variável "categorização dos códigos" explicam $77 \%$ das variações na variável “desfechos”. As medidas de discriminação corroboraram com essa observação, ou seja, a dimensão 1 explica melhor todas as variáveis, tornando mais evidente as relações entre elas.

$\mathrm{Na}$ inspeção visual do gráfico foi possível identificar três grupos de códigos da lista-referência em que há associações entre as categorias de resposta das variáveis, sendo que no $3^{\circ}$ agrupamento não existe representação de nenhuma das categorias de resposta da variável "desfechos" 


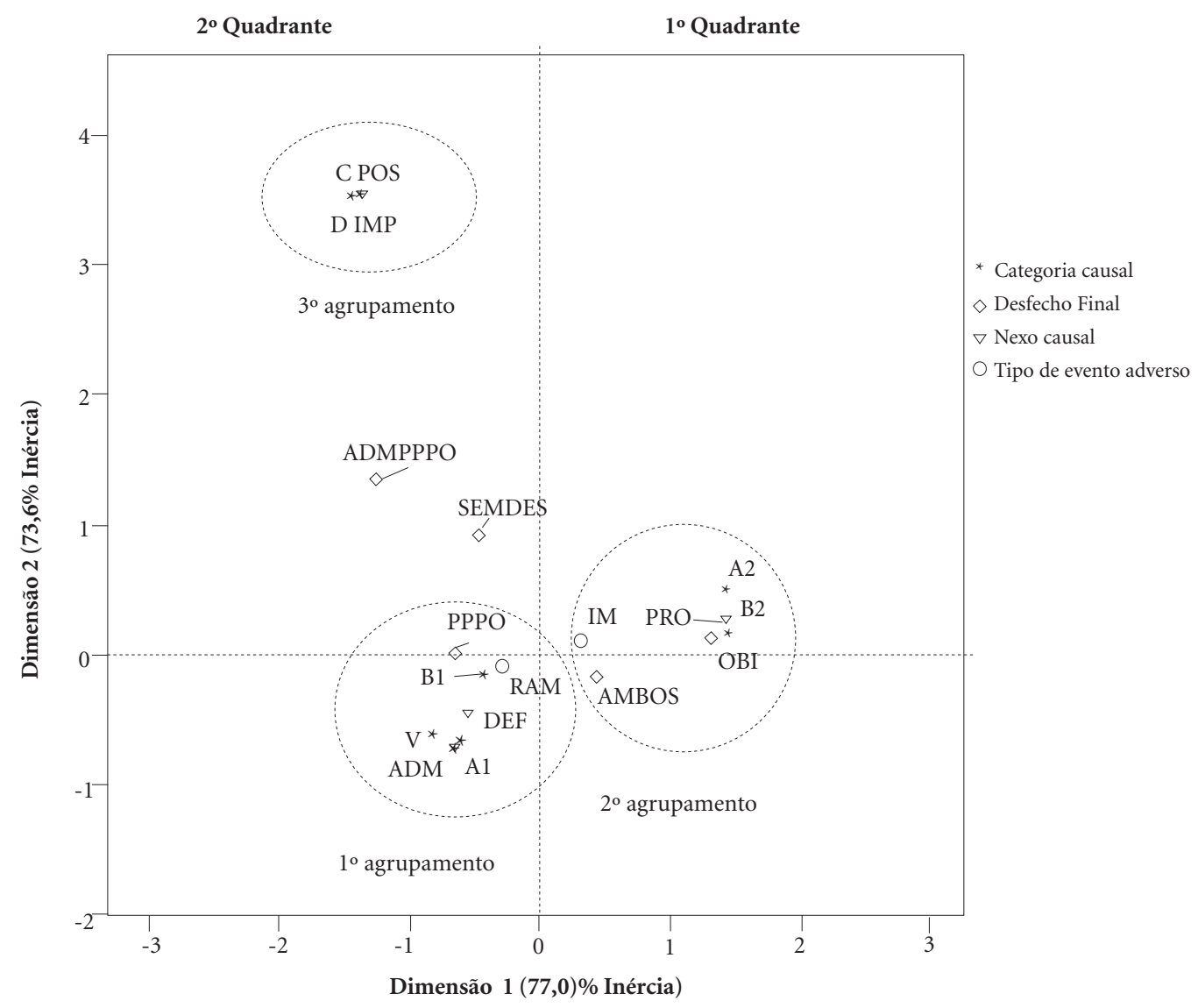

$3^{\circ}$ Quadrante

$4^{\circ}$ Quadrante

Categoria causal: A1, A2, B1, B2, C, D e V. Mais especificações consultar o Quadro 1.

Desfecho final: ADM - Admissão hospitalar; OBI - Óbito; AMBOS - Admissão hospitalar e óbito; ADMPPPO - Admissão hospitalar e pouca possibilidade de produzir óbito; PPPO - Pouca possibilidade de produzir óbito; e SEMDES - Sem produzir nenhum desfecho final.

Nexo causal: DEF - Definido; PRO - Provável; POS - Possível; e IMP - Improvável.

Tipo de evento adverso - RAM - Reações adversas a medicamentos; e IM - Intoxicações medicamentosas.

Figura 2. Mapa de correspondência bidimensional das variáveis estudadas.

e o $2^{\circ}$ agrupamento está no quadrante oposto ao $1{ }^{\circ}$ agrupamento (Figura 2 ).

\section{Discussão}

Os resultados do presente estudo objetivam facilitar a identificação e a quantificação de RAM e IM ao propor uma lista-referência com códigos CID-10 validados e analisados sob diferentes aspectos de interesse à farmacovigilância. Existe uma preocupação crescente das autoridades sanitárias de muitos países em relação à necessidade de diversificação de estratégias para a identificação e quantificação de RAM e IM. A CID-10 é um método eficiente de identificação desses eventos, principalmente naqueles países ou instituições que não têm muitos recursos disponíveis, mas que contam com sistemas de codificação das causas de morbidade e mortalidade confiáveis ${ }^{31}$.

Alguns estudos que utilizaram os códigos da CID-10 não descrevem de forma detalhada como os códigos foram selecionados para a identificação e quantificação de RAM e IM ${ }^{5,8,17}$. Uma exceção corresponde ao estudo de Rozenfeld ${ }^{6}$, cujos requisitos propostos assemelharam-se àqueles definidos na $1^{\text {a }}$ fase desta pesquisa.

Diferentemente do que foi adotado por alguns estudos brasileiros ${ }^{6,8,9}$, os autores desta pesquisa decidiram não suprimir os códigos 
pertencentes as categorias X42, X62 e Y12 por entenderem que outros códigos contidos na lista-referência não permitem também diferenciar os eventos adversos relacionados ao uso de medicamentos daqueles associados ao emprego de outras substâncias lícitas ou não. Além disso, em problemas de saúde que são negligenciados em diferentes aspectos clínicos, epidemiológicos e sanitários por autoridades e profissionais da saúde, a exemplo de RAM e IM, é mais razoável errar por excesso do que por omissão.

O processo de identificação dos códigos para a composição da lista-referência, produziu, na sua grande maioria, códigos que fazem referências explícitas ao envolvimento do medicamento na relação causal, minimizando, de certa forma, a presença de fatores de confundimento nos estudos que porventura utilizarão essa lista. A maioria dos códigos da lista-referência contabilizaram eventos adversos responsáveis por admissão hospitalar e/ou óbito. Esses eventos estão no rol dos adversos graves priorizados nas ações de farmacovigilância realizadas pelas autoridades sanitárias.

Dois capítulos da CID-10 (XIX e XX) concentraram quase $75 \%$ dos códigos relacionados com RAM e IM, sendo que há uma maior similaridade númerica, entre RAM e IM, no capítulo XX. No capítulo XIX (Lesões, envenenamento e algumas outras consequências de causas externas) existe um predomínio dos códigos relacionados com IM. Apesar disso, no estudo publicado por Mota et al. ${ }^{5}$ nenhum código da CID-10 do Capítulo XIX foi encontrado como causa básica de óbito por intoxicação com medicamentos.

Os códigos pertencentes às categorias de resposta A1, B1 e V da variável "Categorização dos códigos”, assim como à categoria de resposta definida da variável "Nexo causal" identificam o medicamento como único agente responsável pela ocorrência do evento adverso, excluído, em sua quase totalidade, fatores de confundimento. $\mathrm{O}$ médico, ao escolher tais códigos, como diagnóstico ou causa básica de óbito, concluiu pela determinação causal medicamento-evento adverso.

Neste estudo, a relação medicamento-evento adverso, definida como RAM, apresentou maior associação aos códigos que registraram casos de admissão hospitalar e aqueles que têm pouca probabilidade de produzirem óbitos. Na relação medicamento-evento adverso, classificada como IM, essa associação foi constatada para códigos que apresentaram, ao mesmo tempo, registros de casos de admissão hospitalar e óbito e, também, para os códigos sem registros de nenhum tipo de desfecho.
Estudos citados por Rodenburg et al. ${ }^{45}$ mostraram que o sexo é um fator de risco importante no uso e resposta aos medicamentos, sendo que as mulheres tendem a ter um maior risco de RAM em comparação com os homens. Essa desigualdade pode ser explicada por diferenças na incidência de problemas de saúde ou pela própria resposta do indivíduo ao medicamento ${ }^{45}$. Em nosso estudo, a lista-referência apresentou um maior número de códigos de uso exclusivo para o sexo feminino em comparação àqueles de exclusividade do sexo masculino. No entanto, a quantidade desses códigos é muito pequena quando comparada ao total de códigos sem restrição ao sexo.

Em vários estudos farmacoepidemiológicos, os pesquisadores, muitas vezes, se interessam em explorar as relações entre (e dentro) um conjunto de variáveis categóricas. Nesta pesquisa, optou-se pela realização do teste ${ }^{2}$ na tabulação cruzada e, também, pela ACM para identificar associações entre variáveis e gerar hipóteses para análises futuras.

A ACM é uma técnica exploratória que fornece uma visão gráfica das relações mais importantes entre variáveis, objetivando identificar as que estão relacionadas entre si e que não são facilmente captadas por inspeção visual de tabulação cruzada ${ }^{46}$. A técnica não exige normalidade para a resposta estudada, ou seja, os testes estatísticos inferencias não são utilizados, estando a solução sugerida pela distribuição dos resultados plotados no gráfico. Variáveis complementares podem ser projetadas sobre as dimensões do gráfico sem interferir nos resultados originais com as variáveis de interesse ${ }^{46}$.

Com base nos resultados das análise das associações de variáveis, algumas tendências gerais podem ser apontadas. Em relação à tabulação cruzada, a variável "Tipo de evento adverso" modificou o efeito do medicamento em relação aos desfechos, tornando a associação entre as variáveis estatisticamente significativa.

Quanto à ACM, verificou-se que o agrupamento 1 é caracterizado pelos códigos em que apresentam o medicamento como único agente causador do evento adverso (A1, B1, V e DEF), estes, por sua vez, estão associados aos códigos que registraram casos de admissão hospitalar (ADM). O agrupamento 2, por outro lado, representa os códigos que citam os medicamentos e outros agentes causadores do evento adverso (A2, B2 e PRO), sendo que estes estão relacionados com os códigos que registraram casos de óbitos (OBI). As categorias de resposta RAM e 
IM da variável "tipo de evento adverso" ficaram mais próximas do agrupamento 1 e 2 , respectivamente.

A principal limitação desta pesquisa diz respeito à utilização da $\mathrm{ACM}$, que, apesar de ser uma técnica estatística útil para identificar grupos relacionados, não revela se tais associações apresentam significância estatística, limitação contornada mediante emprego da tabulação cruzada. Desta forma, é necessária cautela ao interpretar as relações entre as variáveis de linha e coluna no mapa de correspondência. Além disso, tal como em outras técnicas multivariadas, as interpretações dos gráficos são em parte subjetivas, podendo variar de um pesquisador para outro ${ }^{46}$.

Outra limitação diz respeito às deficiências da revisão sistemática utilizada como padrão de referência no estudo que inclui eventos adversos a medicamentos nas mais variadas definições e a não especificidade da lista-referência ${ }^{16}$. No presente estudo, eliminou-se algumas das deficiências, como a inclusão de códigos sobre intoxicação intencional por medicamentos, melhor especificada com a exclusão de códigos das categorias E e U que apresentam pouco ou nenhum envolvimento de medicamentos, não exclusão de códigos relacionados a grupos populacionais e estudos publicados no idioma português ${ }^{5,6,8,9,17}$. As escolhas metodológicas feitas para inclusão de códigos da CID-10 relacionados com RAM e IM pelos autores podem ter ocasionado vieses de seleção e classificação. É importante mencionar que a qualidade dos registros dos códigos CID10 na prática assistencial é um fator que pode influenciar a precisão da lista-referência.

A lista-referência proposta nesta pesquisa pode ser uma ferramenta útil nas ações de farmacovigilância para a vigilância de RAM e IM no Brasil e em países que utilizam a mesma versão brasileira da CID-10. Acredita-se que essa lista facilitará a comparação de estudos farmacoepidemiológicos realizados com dados coletados em distintos momentos do tempo e em diferentes localidades geográficas e instituições de saúde. Tais estudos poderão contribuir com a produção de sinais de alerta de segurança em farmacovigilância.

\section{Colaboradores}

DM Mota, A Vigo e RS Kuchenbecker participaram na concepção e desenho do estudo, análise e interpretação dos dados do manuscrito. DM Mota elaborou a redação do artigo e A Vigo e RS Kuchenbecker participaram da sua revisão crítica e aprovação final. 


\section{Referências}

1. World Health Organization (WHO). Glossary of terms used in Pharmacovigilance. [Internet]. [cited $2014 \mathrm{Nov}$ 15]. Available from: http://www.who-umc.org/graphics/27400.pdf

2. Haerian K, Varn D, Vaidya S, Ena L, Chase H, Friedman C. Detection of Pharmacovigilance-Related adverse Events Using Electronic Health Records and automated Methods. Clin Pharmacol Ther 2013; 92(2):228-234.

3. Goldman SA. Limitations and strengths of spontaneous reports data. Clin Ther 1998; 20(Supl. 3):C40-44.

4. Corrao G. Towards the rational use of Healthcare Utilization Databases for generating real-world evidence new challenges and proposals. Epidemiology, Biostatistics and Public Health 2014; 11(3):e10328.

5. Mota DM, Melo JRR, Freitas DRC, Machado M. Perfil da mortalidade por intoxicação com medicamentos no Brasil, 1996-2005: retrato de uma década. Cien Saude Colet 2012; 17(1):61-70.

6. Rozenfeld S. Agravos provocados por medicamentos em hospitais do Estado do Rio de Janeiro, Brasil. Rev Saude Publica 2007; 41(1):108-115.

7. Carrasco-Garrido P, Andrés LA, Barrera VH, Miguel GA, Jiménez-García R. Trends of adverse drug reactions related-hospitalizations in Spain (2001-2006). BMC Health Serv Res 2010; 10:287.

8. Lessa MDA, Bochner R. Análise das internações hospitalares de crianças menores de um ano relacionadas a intoxicações e efeitos adversos de medicamentos no Brasil. Rev Bras Epidemiol 2008; 11(4):660-674.

9. Paula TC, Bochner R, Montilla DER. Análise clínica e epidemiológica das internações hospitalares de idosos decorrentes de intoxicações e efeitos adversos de medicamentos, Brasil, de 2004 a 2008. Rev Bras Epidemiol 2012; 15(4):828-844.

10. Organização Mundial da Saúde (OMS). Classificação Estatística Internacional de Doenças e Problemas Relacionados à Saúde. 10 ${ }^{\mathrm{a}}$ ed. São Paulo: Editora da USP; 2012.

11. Vougiouklakis T, Boumba VA, Mitselou A. Fatal poisoning in the region of Epirus, Greece, during the period 1998-2004. J Clin Forensic Med 2006; 13(6-8):321325.

12. Uges D. What is the definition of a poisoning? J Clin Forensic Med 2001; 8(1):30-33.

13. World Health Organization (WHO). International Classification of Diseases (ICD) [Internet]. [cited 2015 Feb 16]. Available from: http://www.who.int/classifications/icd/en/

14. Tanno LK, Calderon MA, Goldberg BJ, Akdis CA, Papadopoulos NG, Demoly P. Categorization of allergic disorders in the new World Health Organization International Classification of Diseases. Clin $\operatorname{Tr} 2014$; 4(42):1-8.

15. Gerstman B. Case definition and disease classification. In: Gerstman B, editor. Epidemiology Kept Simple: An Introduction to Traditional and Modern Epidemiology. $3^{\text {th }}$ ed. Chichester: John Wiley \& Sons Ltd; 2013. p. 347354.

16. Hohl CM, Karpov A, Reddekopp L, Stausberg J. ICD-10 codes used to identify adverse drug events in administrative data: a systematic review. J Am Med Inform Assoc 2014; 21(3):547-557.
17. Tanno LK, Ganem F, Demoly P, Toscano CM, Bierrenbach AL. Undernotification of anaphylaxis deaths in Brazil due to difficult coding under the ICD-10. Allergy Eur J Allergy Clin Immunol 2012; 67(6):783-789.

18. Hoyert DL, Xu J. Deaths: preliminary data for 2011. Natl Vital Stat Rep 2012; 61(6):1-51.

19. Okumura Y, Shimizu S, Ishikawa KB, Matsuda S, Fushimi $\mathrm{K}$, Ito $\mathrm{H}$. Comparison of emergency hospital admissions for drug poisoning and major diseases: a retrospective observational study using a nationwide administrative discharge database. BMJ Open 2012; 2(6):e001857.

20. Ossiander EM. Using textual cause-of-death data to study drug poisoning deaths. Am J Epidemiol 2014; 179(7):884-894.

21. Stausberg J. International prevalence of adverse drug events in hospitals: an analysis of routine data from England, Germany, and the USA. BMC Health Serv Res 2014; 14:125.

22. Stausberg J, Hasford J. Drug-related admissions and hospital-acquired adverse drug events in Germany: a longitudinal analysis from 2003 to 2007 of ICD-10coded routine data. BMC Health Serv Res 2011; 11:134.

23. Stausberg J, Hasford J. Identification of adverse drug events: the use of ICD-10 coded diagnoses in routine hospital data. Dtsch Arztebl Int 2010; 107(3):23-29.

24. Regev A, Seeff LB, Merz M, Ormarsdottir S, Aithal GP, Gallivan J, Watkins PB. Causality Assessment for Suspected DILI During Clinical Phases of Drug Development. Drug Saf 2014; 37(1):47-56.

25. Fontana RJ, Watkins PB, Bonkovsky HL, Chalasani N, Davern T, Serrano J, Rochon J; DILIN Study Group. Drug-induced liver injury network (DILIN) prospective study: Rationale, design and conduct. Drug Saf 2009; 32(1):55-68.

26. World Health Organization (WHO). The use of the WHO-UMC system for standardized case causality assessment. Uppsala: The Uppsala Monitoring Centre; 2005.

27. Doey L, Kurta J. Correspondence analysis applied to psychological research. Tutor Quant Methods Psychol 2011; 7(1):5-14.

28. Li G, Warner M, Lang BH, Huang L, Sun LS. Epidemiology of anesthesia-related mortality in the United States, 1999-2005. Anesthesiology 2009; 110(4):759-765.

29. Barrow P, Waller P, Wise L. Comparison of hospital episodes with "drug-induced" disorders and spontaneously reported adverse drug reactions. Br J Clin Pharmacol 2006; 61(2):233-237.

30. Elalamy I, Le Gal G, Nachit-Ouinekh F, Lafuma A, Emery C, Le-Fur C, Chapuis F. Heparin-induced thrombocytopenia: an estimate of the average cost in the hospital setting in France. Clin Appl Thromb Hemost 2009; 15(4):428-434.

31. Hodgkinson MR, Dirnbauer NJ, Larmour I. Identification of adverse drug reactions using the ICD-10 Australian Modification Clinical Coding Surveillance. J Pharm Pract Res 2009; 39(1):19-23.

32. Lyytikäinen $\mathrm{O}$, Turunen $\mathrm{H}$, Sund $\mathrm{R}$, Rasinperä $\mathrm{M}$, Könönen E, Ruutu P, Keskimäki I. Hospitalizations and deaths associated with clostridium difficile infection, Finland, 1996-2004. Emerg Infect Dis 2009; 15(5):761-765. 
33. Patel H, Bell D, Molokhia M, Srishanmuganathan J, Patel M, Car J, Majeed A. Trends in hospital admissions for adverse drug reactions in England: analysis of national hospital episode statistics 1998-2005. BMC Clin Pharmacol 2007; 7:9.

34. Runciman WB, Roughead EE, Semple SJ, Adams RJ. Adverse drug events and medication errors in Australia. Int J Qual Health Care 2003; 15(Supl. 1):i49-59.

35. Shepherd G, Mohorn P, Yacoub K, May DW. Adverse drug reaction deaths reported in United States vital statistics, 1999-2006. Ann Pharmacother 2012; 46(2):169175.

36. Sims SA, Snow LA, Porucznik CA. Surveillance of methadone-related adverse drug events using multiple public health data sources. J Biomed Inform 2007; 40(4):382-389.

37. Waller P, Shaw M, Ho D, Shakir S, Ebrahim S. Hospital admissions for "drug-induced" disorders in England: A study using the Hospital Episodes Statistics (HES) database. Br J Clin Pharmacol 2005; 59(2):213-219.

38. Wu T-Y, Jen M-H, Bottle A, Molokhia M, Aylin P, Bell $D$, Majeed A. Ten-year trends in hospital admissions for adverse drug reactions in England 1999-2009. J R Soc Med 2010; 103(6):239-250.

39. Wysowski DK, Swartz L, Borders-Hemphill BV, Goulding MR, Dormitzer C. Use of parenteral iron products and serious anaphylactic-type reactions. Am J Hematol 2010; 85(9):650-654.

40. Wysowski DK. Surveillance of prescription drug-related mortality using death certificate data. Drug Saf 2007; 30(6):533-540.

41. Wysowski DK, Nourjah P. Letter to the Editor Analyzing prescription drugs as causes. Public Health Rep 2004; 119(December):2004.
42. Wysowski DK, Nourjah P. Deaths attributed to X-ray contrast media on U.S. death certificates. Am J Roentgenol 2006; 186(3):613-615.

43. Zhang M, Holman CDJ, Price SD, Sanfilippo FM, Preen DB, Bulsara MK. Comorbidity and repeat admission to hospital for adverse drug reactions in older adults: retrospective cohort study. BMJ 2009; 338:a2752.

44. Zhang M, Holman CDAJ, Preen DB, Brameld K. Repeat adverse drug reactions causing hospitalization in older Australians: A population-based longitudinal study 1980-2003. Br J Clin Pharmacol 2007; 63(2):163-170.

45. Rodenburg EM, Stricker BHC, Visser LE. Sex-related differences in hospital admissions attributed to adverse drug reactions in the Netherlands. Br J Clin Pharmacol 2011; 71(1):95-104.

46. Sourial N, Wolfson C, Zhu B, Quail J, Fletcher J, Karunananthan S, Bandeen-Roche K, Béland F, Bergman H. Correspondence analysis is a useful tool to uncover the relationships among categorical variables. $J$ Clin Epidemiol 2010; 63(6):638-646.

Artigo apresentado em 03/09/2015

Aprovado em 01/10/2016

Versão final apresentada em 03/10/2016 